\title{
The Dirichlet problem for the Laplace equation in supershaped annuli
}

\author{
Diego Caratelli ${ }^{1}$, Johan Gielis ${ }^{2 *}$, Ilia Tavkhelidze ${ }^{3}$ and Paolo E Ricci ${ }^{4}$
}

"Correspondence:

johan.gielis@ua.ac.be

${ }^{2}$ Department of Bioscience

Engineering, University of Antwerp,

Antwerp, Belgium

Full list of author information is

available at the end of the article

\begin{abstract}
The Dirichlet problem for the Laplace equation in normal-polar annuli is addressed by using a suitable Fourier-like technique. Attention is in particular focused on the wide class of domains whose boundaries are defined by the so-called 'superformula' introduced by Gielis. A dedicated numerical procedure based on the computer algebra system Mathematica ${ }^{\odot}$ is developed in order to validate the proposed methodology. In this way, highly accurate approximations of the solution, featuring properties similar to the classical ones, are obtained.
\end{abstract}

\section{Introduction}

Many problems of mathematical physics and electromagnetics are related to the Laplacian [1]. In recent papers [2-9], the classical Fourier projection method [10, 11] for solving boundary-value problems ( $B V P \mathrm{~s})$ for the Laplace and Helmholtz equations in canonical domains has been extended in order to address similar differential problems in simply connected starlike domains, whose boundaries may be regarded as an anisotropically stretched unit circle centered at the origin.

In this contribution, a suitable technique useful to compute the coefficients of the Fourier-like expansion representing the solution of the Dirichlet boundary-value problem for the Laplace equation in complex annular domains is presented. In particular, the boundaries of the considered domains are supposed to be defined by the so-called Gielis formula [12]. Regular functions are assumed to describe the boundary values, but the proposed approach can be easily generalized in the case of weakened hypotheses. In order to verify and validate the developed methodology, a suitable numerical procedure based on the computer algebra system Mathematica ${ }^{\odot}$ has been adopted. By using such a procedure, a point-wise convergence of the Fourier-like series representation of the solution has been observed in the regular points of the boundaries, with Gibbs-like phenomena potentially occurring in the quasi-cusped points. The obtained numerical results are in good agreement with theoretical findings by Carleson [13].

\section{The Laplacian in stretched polar coordinates}

Let us introduce in the real plane the usual polar coordinate system

$$
\left\{\begin{array}{l}
x=r \cos \vartheta \\
y=r \sin \vartheta
\end{array}\right.
$$


and the polar equations

$$
r=R_{ \pm}(\vartheta)
$$

relevant to the boundaries of the supershaped annulus $\mathcal{A}$ which is described by the following chain of inequalities:

$$
R_{-}(\vartheta) \leq r \leq R_{+}(\vartheta),
$$

with $0 \leq \vartheta \leq 2 \pi$. In (2) $R_{ \pm}(\vartheta)$ are assumed to be piece-wise $C^{2}$ functions satisfying the condition

$$
R_{+}(\vartheta)>R_{-}(\vartheta)>0, \quad 0 \leq \vartheta \leq 2 \pi .
$$

In this way, upon introducing the stretched radius $\varrho$ such that

$$
r=\frac{(b-\varrho) R_{-}(\vartheta)-(a-\varrho) R_{+}(\vartheta)}{b-a},
$$

with $b>a>0$, the considered annular domain $\mathcal{A}$ can be readily obtained by assuming $0 \leq \vartheta \leq 2 \pi$ and $a \leq \varrho \leq b$.

Remark Note that in the stretched coordinate system $\varrho, \vartheta$, the original domain $\mathcal{A}$ is transformed into the circular annulus of radii $a$ and $b$, respectively. Hence, in this system one can use classical techniques to solve the Laplace equation, including the eigenfunction method [11].

Let us consider a piece-wise $C^{2}(\mathcal{A})$ function $v(x, y)=v(r \cos \vartheta, r \sin \vartheta)=u(r, \vartheta)$ and the Laplace operator in polar coordinates

$$
\Delta u=\frac{\partial^{2} u}{\partial r^{2}}+\frac{1}{r} \frac{\partial u}{\partial r}+\frac{1}{r^{2}} \frac{\partial^{2} u}{\partial \vartheta^{2}} .
$$

In the considered stretched coordinate system $\Delta$ can be represented by setting

$$
U(\varrho, \vartheta)=u\left(\frac{(b-\varrho) R_{-}(\vartheta)-(a-\varrho) R_{+}(\vartheta)}{b-a}, \vartheta\right)
$$

In this way, by denoting $R_{ \pm}(\vartheta)$ as $R_{ \pm}$for the sake of shortness, one can readily find

$$
\begin{aligned}
\frac{\partial u}{\partial r}= & \frac{b-a}{R_{+}-R_{-}} \frac{\partial U}{\partial \varrho}, \\
\frac{\partial^{2} u}{\partial r^{2}}= & \left(\frac{b-a}{R_{+}-R_{-}}\right)^{2} \frac{\partial^{2} U}{\partial \varrho^{2}}, \\
\frac{\partial^{2} u}{\partial \vartheta^{2}}= & \left\{\frac{(b-\rho)\left[2 \dot{R}_{-}\left(\dot{R}_{+}-\dot{R}_{-}\right)-\ddot{R}_{-}\left(R_{+}-R_{-}\right)\right]}{\left(R_{+}-R_{-}\right)^{2}}\right. \\
& \left.-\frac{(a-\rho)\left[2 \dot{R}_{+}\left(\dot{R}_{+}-\dot{R}_{-}\right)-\ddot{R}_{+}\left(R_{+}-R_{-}\right)\right]}{\left(R_{+}-R_{-}\right)^{2}}\right\} \frac{\partial U}{\partial \varrho} \\
& +\left[\frac{(b-\varrho) \dot{R}_{-}-(a-\varrho) \dot{R}_{+}}{R_{+}-R_{-}}\right]^{2} \frac{\partial^{2} U}{\partial \varrho^{2}}-2 \frac{(b-\varrho) \dot{R}_{-}-(a-\varrho) \dot{R}_{+}}{R_{+}-R_{-}} \frac{\partial^{2} U}{\partial \varrho \partial \vartheta}+\frac{\partial^{2} U}{\partial \vartheta^{2}},
\end{aligned}
$$


where the dot superscript denotes the differentiation with respect to the angle $\vartheta$. Substituting equations (8)-(10) into equation (6) finally yields

$$
\begin{aligned}
\Delta u= & \left(\frac{b-a}{R_{+}-R_{-}}\right)^{2}\left(\left\{1+\left[\frac{(b-\varrho) \dot{R}_{-}-(a-\varrho) \dot{R}_{+}}{(b-\varrho) R_{-}-(a-\varrho) R_{+}}\right]^{2}\right\} \frac{\partial^{2} U}{\partial \varrho^{2}}\right. \\
& +\left\{\frac{(b-\rho)\left[\left(R_{-}-\ddot{R}_{-}\right)\left(R_{+}-R_{-}\right)+2 \dot{R}_{-}\left(\dot{R}_{+}-\dot{R}_{-}\right)\right]}{\left[(b-\varrho) R_{-}-(a-\varrho) R_{+}\right]^{2}}\right. \\
& \left.-\frac{(a-\rho)\left[\left(R_{+}-\ddot{R}_{+}\right)\left(R_{+}-R_{-}\right)+2 \dot{R}_{+}\left(\dot{R}_{+}-\dot{R}_{-}\right)\right]}{\left[(b-\varrho) R_{-}-(a-\varrho) R_{+}\right]^{2}}\right\} \frac{\partial U}{\partial \varrho} \\
& -2\left(R_{+}-R_{-}\right) \frac{(b-\varrho) \dot{R}_{-}-(a-\varrho) \dot{R}_{+}}{\left[(b-\varrho) R_{-}-(a-\varrho) R_{+}\right]^{2}} \frac{\partial^{2} U}{\partial \varrho \partial \vartheta} \\
& \left.+\left[\frac{R_{+}-R_{-}}{(b-\varrho) R_{-}-(a-\varrho) R_{+}}\right]^{2} \frac{\partial^{2} U}{\partial \vartheta^{2}}\right) .
\end{aligned}
$$

As it can be easily noticed, upon setting $R_{-}(\vartheta)=a=0$ and $R_{+}(\vartheta)=b=1$, the classical expression of the Laplacian in polar coordinates is recovered.

\section{The Dirichlet problem for the Laplace equation}

Let us consider the interior Dirichlet problem for the Laplace equation in a starlike annulus $\mathcal{A}$, whose boundaries $\partial_{ \pm} \mathcal{A}$ are described by the polar equations $r=R_{ \pm}(\vartheta)$ respectively

$$
\begin{cases}\Delta v(x, y)=0, & (x, y) \in \stackrel{\mathcal{A}}{ } \\ v(x, y)=f_{ \pm}(x, y), & (x, y) \in \partial_{ \pm} \mathcal{A} .\end{cases}
$$

Under the mentioned assumptions, one can prove the following theorem.

\section{Theorem Let}

$$
f_{ \pm}\left(R_{ \pm}(\vartheta) \cos \vartheta, R_{ \pm}(\vartheta) \sin \vartheta\right)=F_{ \pm}(\vartheta)=\sum_{m=0}^{+\infty}\left(\alpha_{m}^{( \pm)} \cos m \vartheta+\beta_{m}^{( \pm)} \sin m \vartheta\right)
$$

where

$$
\left\{\begin{array}{c}
\alpha_{m}^{( \pm)} \\
\beta_{m}^{( \pm)}
\end{array}\right\}=\frac{\epsilon_{m}}{2 \pi} \int_{0}^{2 \pi} F_{ \pm}(\vartheta)\left\{\begin{array}{c}
\cos m \vartheta \\
\sin m \vartheta
\end{array}\right\} d \vartheta
$$

$\epsilon_{m}$ being the usual Neumann symbol. Then the boundary-value problem (12) for the Laplace equation admits a classical solution $v(x, y) \in L^{2}(\mathcal{A})$ such that the following Fourierlike series expansion holds true:

$$
\begin{aligned}
v( & \left(\frac{(b-\varrho) R_{-}(\vartheta)-(a-\varrho) R_{+}(\vartheta)}{b-a} \cos \vartheta, \frac{(b-\varrho) R_{-}(\vartheta)-(a-\varrho) R_{+}(\vartheta)}{b-a} \sin \vartheta\right) \\
= & U(\varrho, \vartheta) \\
= & \sum_{m=-\infty}^{+\infty}\left[\frac{(b-\varrho) R_{-}(\vartheta)-(a-\varrho) R_{+}(\vartheta)}{b-a}\right]^{m}\left(A_{m} \cos m \vartheta+B_{m} \sin m \vartheta\right) \\
& \quad+\delta_{0} \ln \left(\frac{(b-\varrho) R_{-}(\vartheta)-(a-\varrho) R_{+}(\vartheta)}{b-a}\right) .
\end{aligned}
$$


For each index $m$, define

$$
\left[\begin{array}{l}
\xi_{m}^{( \pm)}(\vartheta) \\
\eta_{m}^{( \pm)}(\vartheta)
\end{array}\right]=R_{ \pm}(\vartheta)\left[\begin{array}{c}
\cos m \vartheta \\
\sin m \vartheta
\end{array}\right],
$$

and set, for shortness, $\zeta^{( \pm)}(\vartheta)=\ln R_{ \pm}(\vartheta)$. In this way, the coefficients $\delta_{0}$ and $A_{m}, B_{m}$ appearing in (15) can be determined by solving the infinite linear system

$$
\sum_{m=-\infty}^{+\infty}\left[\begin{array}{cc}
\mathrm{X}_{\mathrm{C}_{n, m}}^{(-)} & \mathrm{Y}_{\mathrm{C}_{n, m}}^{(-)} \\
\mathrm{X}_{\mathrm{S}_{n, m}}^{(-)} & \mathrm{Y}_{\mathrm{S}_{n, m}}^{(-)} \\
\mathrm{X}_{\mathrm{C}_{n, m}}^{(+)} & \mathrm{Y}_{\mathrm{C}_{n, m}}^{(+)} \\
\mathrm{X}_{\mathrm{S}_{n, m}}^{(+)} & \mathrm{Y}_{\mathrm{S}_{n, m}}^{(+)}
\end{array}\right] \cdot\left[\begin{array}{c}
A_{m} \\
B_{m}
\end{array}\right]+\left[\begin{array}{c}
\mathrm{Z}_{\mathrm{C}_{n}}^{(-)} \\
\mathrm{Z}_{\mathrm{S}_{n}}^{(-)} \\
\mathrm{Z}_{\mathrm{C}_{n}}^{(+)} \\
\mathrm{Z}_{\mathrm{S}_{n}}^{(+)}
\end{array}\right] \delta_{0}=\left[\begin{array}{c}
\alpha_{n}^{(-)} \\
\beta_{n}^{(-)} \\
\alpha_{n}^{(+)} \\
\beta_{n}^{(+)}
\end{array}\right],
$$

where

$$
\begin{aligned}
& \mathrm{X}_{\left\{\begin{array}{l}
\mathrm{C} \\
\mathrm{S}
\end{array}\right\}_{n, m}^{( \pm)}}=\frac{\epsilon_{n}}{2 \pi} \int_{0}^{2 \pi} \xi_{m}^{( \pm)}(\vartheta)\left\{\begin{array}{c}
\cos n \vartheta \\
\sin n \vartheta
\end{array}\right\} d \vartheta \\
& \mathrm{Y}_{\left\{\begin{array}{l}
\mathrm{C} \\
\mathrm{S}\}_{n, m}
\end{array}\right.}^{( \pm)}=\frac{\epsilon_{n}}{2 \pi} \int_{0}^{2 \pi} \eta_{m}^{( \pm)}(\vartheta)\left\{\begin{array}{c}
\cos n \vartheta \\
\sin n \vartheta
\end{array}\right\} d \vartheta \\
& \mathrm{Z}_{\left\{\begin{array}{l}
\mathrm{C} \\
\mathrm{S}\}_{n}
\end{array}\right.}^{( \pm)}=\frac{\epsilon_{n}}{2 \pi} \int_{0}^{2 \pi} \zeta^{( \pm)}(\vartheta)\left\{\begin{array}{l}
\cos n \vartheta \\
\sin n \vartheta
\end{array}\right\} d \vartheta,
\end{aligned}
$$

with $m \in \mathbb{Z}$, and $n \in \mathbb{N}_{0}:=\mathbb{N} \cup\{0\}$.

Proof Upon noting that in the stretched coordinate system $\varrho, \vartheta$ introduced in the $x, y$ plane, the considered domain $\mathcal{A}$ turns into the circular annulus of radii $a$ and $b$, one can readily adopt the usual eigenfunction method [11] in combination with the separation of variables (with respect to $r$ and $\vartheta$ ). As a consequence, elementary solutions of the problem can be searched in the form

$$
u(r, \vartheta)=U\left(\frac{b\left[r-R_{-}(\vartheta)\right]-a\left[r-R_{+}(\vartheta)\right]}{R_{+}(\vartheta)-R_{-}(\vartheta)}, \vartheta\right)=\mathrm{P}(r) \Theta(\vartheta) .
$$

Substituting into the Laplace equation, one easily finds that the functions $P(\cdot), \Theta(\cdot)$ must satisfy the ordinary differential equations

$$
\begin{aligned}
& \frac{d^{2} \Theta(\vartheta)}{d \vartheta^{2}}+\mu^{2} \Theta(\vartheta)=0, \\
& r^{2} \frac{d^{2} \mathrm{P}(r)}{d r^{2}}+r \frac{d \mathrm{P}(r)}{d r}-\mu^{2} \mathrm{P}(r)=0,
\end{aligned}
$$

respectively. The parameter $\mu$ is a separation constant whose choice is governed by the physical requirement that at any fixed point in the real plane the scalar field $u(r, \vartheta)$ must be single-valued. So, by setting $\mu=m \in \mathbb{N}_{0}$, one can easily find

$$
\Theta(\vartheta)=a_{m} \cos m \vartheta+b_{m} \sin m \vartheta,
$$


where $a_{m}, b_{m} \in \mathbb{C}$ denote arbitrary constants. The radial function $\mathrm{P}(\cdot)$ satisfying (23) can be readily expressed as follows:

$$
\mathrm{P}(r)= \begin{cases}c_{m} r^{m}+d_{m} r^{-m}, & m \neq 0, \\ c_{0}+d_{0} \ln r, & m=0,\end{cases}
$$

with $c_{m}, d_{m} \in \mathbb{C}$. Therefore, the general solution of the Dirichlet problem (12) can be searched in the form

$$
u(r, \vartheta)=\sum_{m=-\infty}^{+\infty} r^{m}\left(A_{m} \cos m \vartheta+B_{m} \sin m \vartheta\right)+\delta_{0} \ln r
$$

Enforcing the Dirichlet boundary condition readily yields $F_{ \pm}(\vartheta)=u\left(R_{ \pm}(\vartheta), \vartheta\right)$. Hence, using the classical Fourier projection method, equations (17)-(20) follow after some trivial manipulations.

It is worth noting that the derived expressions still hold under the assumption that $R_{ \pm}(\vartheta)$ are piecewise continuous functions, and the boundary values are described by square integrable, not necessarily continuous, functions so that the relevant Fourier coefficients $\alpha_{m}^{( \pm)}$, $\beta_{m}^{( \pm)}$in equation (14) are finite quantities.

\section{Numerical procedure}

In the following numerical examples, let us assume, for the boundaries $\partial_{ \pm} \mathcal{A}$ of the considered annulus, general polar equations of the type

$$
R_{ \pm}(\vartheta)=\left(\left|\frac{1}{d_{x}^{ \pm}} \cos \frac{k_{x}^{ \pm} \vartheta}{4}\right|^{v_{x}^{ \pm}}+\left|\frac{1}{d_{y}^{ \pm}} \sin \frac{k_{y}^{ \pm} \vartheta}{4}\right|^{v_{y}^{ \pm}}\right)^{-1 / \nu_{0}^{ \pm}}
$$

as introduced by Gielis in [12]. Very different characteristic geometries, including ellipses, Lamé curves, ovals, and $m$-fold symmetric figures are obtained by assuming suitable values of the parameters $k_{x}^{ \pm}, k_{y}^{ \pm}, d_{x}^{ \pm}, d_{y}^{ \pm}, v_{x}^{ \pm}, v_{y}^{ \pm}, v_{0}^{ \pm}$in (27). It is emphasized that almost all two-dimensional normal-polar annular domains can be described, or closely approximated, by (27).

In order to assess the performance of the proposed methodology in terms of numerical accuracy and convergence rate, the relative boundary error has been evaluated as follows:

$$
e_{N}=\frac{\left\|U_{N}(a, \vartheta)-F_{-}(\vartheta)\right\|}{\left\|F_{-}(\vartheta)\right\|}+\frac{\left\|U_{N}(b, \vartheta)-F_{+}(\vartheta)\right\|}{\left\|F_{+}(\vartheta)\right\|},
$$

with $\|\cdot\|$ being the usual $L^{2}$ norm, and where $U_{N}(\varrho, \vartheta)$ denotes the partial sum of order $N$ relevant to the Fourier-like series expansion representing the solution of the boundaryvalue problem for the Laplace equation, namely

$$
\begin{aligned}
U_{N}(\varrho, \vartheta)= & \sum_{m=-N}^{N}\left[\frac{(b-\varrho) R_{-}(\vartheta)-(a-\varrho) R_{+}(\vartheta)}{b-a}\right]^{m}\left(A_{m} \cos m \vartheta+B_{m} \sin m \vartheta\right) \\
& +\delta_{0} \ln \left(\frac{(b-\varrho) R_{-}(\vartheta)-(a-\varrho) R_{+}(\vartheta)}{b-a}\right) .
\end{aligned}
$$


Figure 1 Relative boundary error $e_{N}$ as a function of the order $N$ of the truncated Fourier-like series expansion representing the solution of the Dirichlet problem for the Laplace equation in the supershaped annulus $\mathcal{A}$ described by the Gielis formula with parameters $k_{x}^{ \pm}=k_{y}^{ \pm}=3, d_{x}^{-}=d_{y}^{-}=3 / 4, d_{x}^{+}=d_{y}^{+}=5 / 2, v_{x}^{ \pm}=v_{y}^{ \pm}$ $=12, v_{0}^{ \pm}=21$.

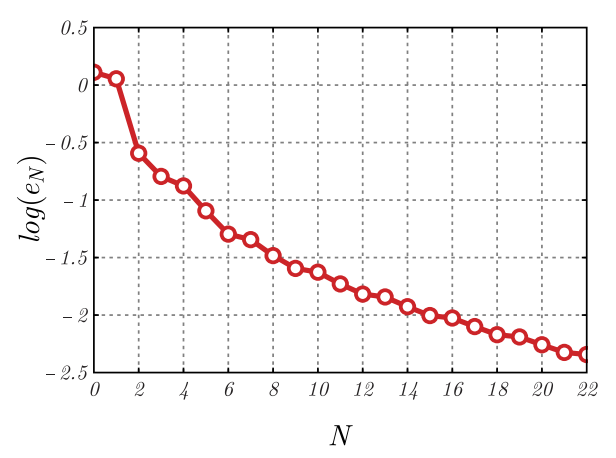

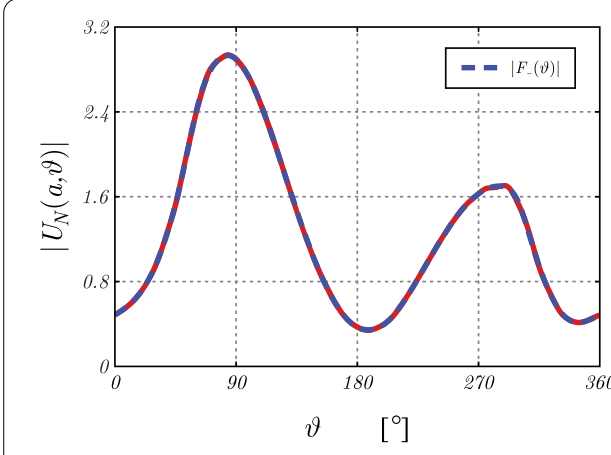

(a)

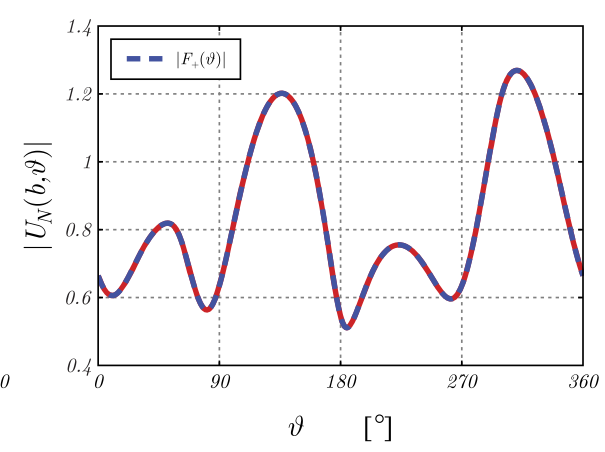

(b)

Figure 2 Boundary behavior along $\partial_{-} \mathcal{A}(a)$ and $\partial_{+} \mathcal{A}(b)$ of the partial sum $U_{N}(\varrho, \vartheta)$ of order $N=22$ representing the solution of the Dirichlet problem for the Laplace equation in the supershaped annulus $\mathcal{A}$ described by the Gielis formula with parameters $k_{x}^{ \pm}=k_{y}^{ \pm}=3, d_{x}^{-}=d_{y}^{-}=3 / 4, d_{x}^{+}=d_{y}^{+}=5 / 2$, $v_{x}^{ \pm}=v_{y}^{ \pm}=12, v_{0}^{ \pm}=21$.

Remark It is to be noticed that where the boundary values exhibit a rapidly oscillating behavior, the order $N$ of the expansion (29) approximating the solution of the problem should be increased accordingly in order to achieve the desired numerical accuracy.

\section{First example}

By assuming in (27) $k_{x}^{ \pm}=k_{y}^{ \pm}=3, d_{x}^{-}=d_{y}^{-}=3 / 4, d_{x}^{+}=d_{y}^{+}=5 / 2, v_{x}^{ \pm}=v_{y}^{ \pm}=12, v_{0}^{ \pm}=21$, the annulus $\mathcal{A}$ features a triangular strip-like shape. Let $f_{-}(x, y)=e^{-x^{2}+y}+2 i y^{2}$ and $f_{+}(x, y)=$ $\cos \frac{x+y}{2}+i \sin \frac{x y}{2}$ be the functions describing the boundary values. Under these assumptions, the relative boundary error $e_{N}$ as a function of the number $N$ of terms in the truncated series expansion (29) exhibits the behavior shown in Figure 1. As it appears from Figure 2, the selection of the expansion order $N=22$ leads to a very accurate Fourier-like representation $v_{N}(x, y)$ of the solution (featuring boundary error $e_{N}<0.5 \%$ ). The spatial distribution of $v_{N}(x, y)$ is shown in Figure 3, whereas the magnitude and phase of the relevant Fourier expansion coefficients $A_{m}$ and $B_{m}(|m| \leq N)$ are plotted in Figure 4.

\section{Second example}

In the second numerical example, we turn to the consideration of the class of annuli having one or both boundaries featuring a polygonal contour. In this respect, it is not difficult to show that the general $k$-sided convex regular polygon can be readily described by the 
Figure 3 Spatial distribution of the Fourier-like series expansion $v_{N}(x, y)$ of order $N=22$ representing the solution of the Dirichlet problem for the Laplace equation in the supershaped annulus $\mathcal{A}$ described by the Gielis formula with parameters $k_{x}^{ \pm}=k_{y}^{ \pm}=3, d_{x}^{-}=d_{y}^{-}$ $=3 / 4, d_{x}^{+}=d_{y}^{+}=5 / 2, v_{x}^{ \pm}=v_{y}^{ \pm}=12, v_{0}^{ \pm}=21$.

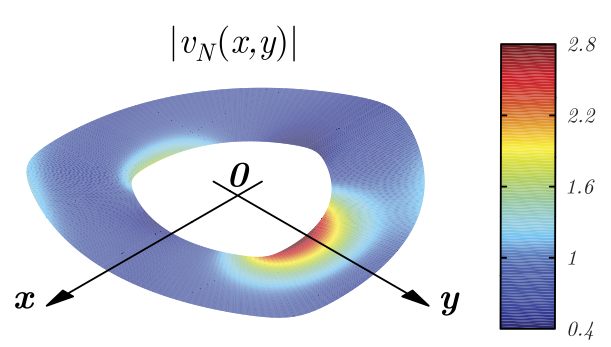

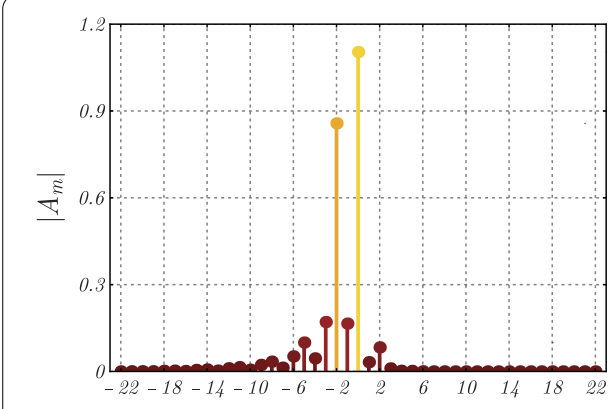

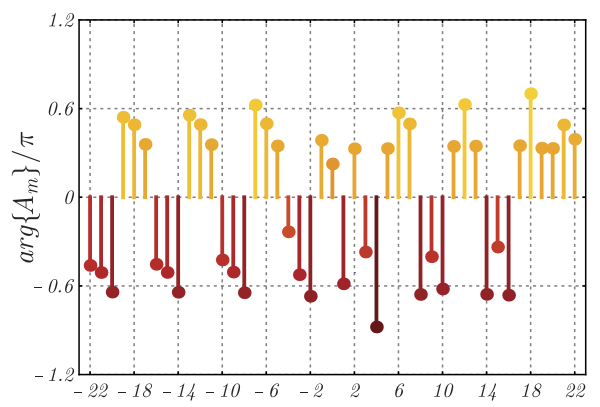

$m$

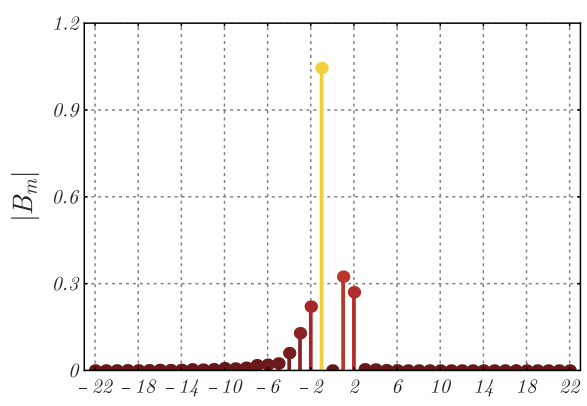

(b)

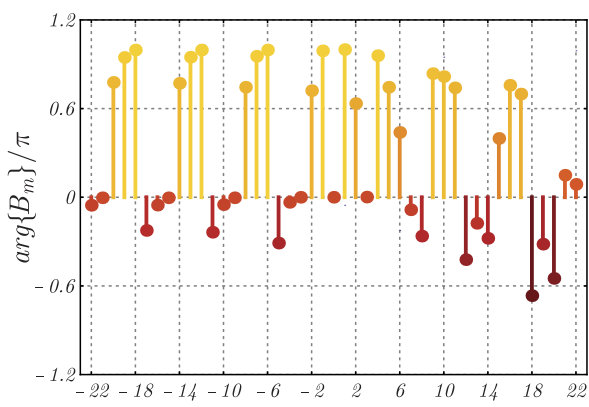

(d)

Figure 4 Magnitude ( $a$ ), (b) and phase (c), (d) of the coefficients $A_{m}$ and $B_{m}$ relevant to the expansion $v_{N}(x, y)$ of order $N=22$ representing the solution of the Dirichlet problem for the Laplace equation in the supershaped annulus $\mathcal{A}$ described by the Gielis formula with parameters $k_{x}^{ \pm}=k_{y}^{ \pm}=3$, $d_{x}^{-}=d_{y}^{-}=3 / 4, d_{x}^{+}=d_{y}^{+}=5 / 2, v_{x}^{ \pm}=v_{y}^{ \pm}=12, v_{0}^{ \pm}=21$.

following specialized version of Gielis' formula [14]:

$$
R_{k}(\vartheta)=\lim _{\nu \rightarrow+\infty}\left[\left|\frac{1}{d} \cos \frac{k \vartheta}{4}\right|^{2\left(1-\nu \log _{2} \cos \frac{\pi}{k}\right)}+\left|\frac{1}{d} \sin \frac{k \vartheta}{4}\right|^{2\left(1-\nu \log _{2} \cos \frac{\pi}{k}\right)}\right]^{-1 / \nu} .
$$

In this way, the methodology detailed in the previous section can be used straightforwardly. In particular, upon assuming in (27) $k_{x}^{-}=k_{y}^{-}=2, d_{x}^{-}=1 / 2, d_{y}^{-}=1, v_{x}^{-}=2, v_{y}^{-}=$ $v_{0}^{-}=4$, as well as $k_{x}^{+}=k_{y}^{+}=k^{+}=4, d_{x}^{+}=d_{y}^{+}=7 / 4$, and $v_{x}^{+}=v_{y}^{+}=2\left(1-v_{0}^{+} \log _{2} \cos \frac{\pi}{k^{+}}\right)$, with $v_{0}^{+} \rightarrow+\infty$, the annulus $\mathcal{A}$ may be regarded as the result of the Boolean subtraction of an ovaloid from a square. Let $f_{-}(x, y)=\sinh (x+y)+i \cosh (x y)$ and $f_{+}(x, y)=1$ be the functions describing the boundary values along $\partial_{\mp} \mathcal{A}$, respectively. Under these assumptions, the relative boundary error $e_{N}$ exhibits the behavior shown in Figure 5. As it appears from 
Figure 5 Relative boundary error $e_{N}$ as a function of the order $N$ of the truncated Fourier-like series expansion representing the solution of the Dirichlet problem for the Laplace equation in the supershaped annulus $\mathcal{A}$ described by the Gielis formula with parameters $k_{x}^{-}=k_{y}^{-}=2, d_{x}^{-}=1 / 2, d_{y}^{-}=1, v_{x}^{-}=2, v_{y}^{-}=v_{0}^{-}=4$, $k_{x}^{+}=k_{y}^{+}=k^{+}=4, d_{x}^{+}=d_{y}^{+}=7 / 4$, and $v_{x}^{+}=v_{y}^{+}=2(1$ $\left.-v_{0}^{+} \log _{2} \cos \frac{\pi}{k^{+}}\right), v_{0}^{+} \rightarrow+\infty$.
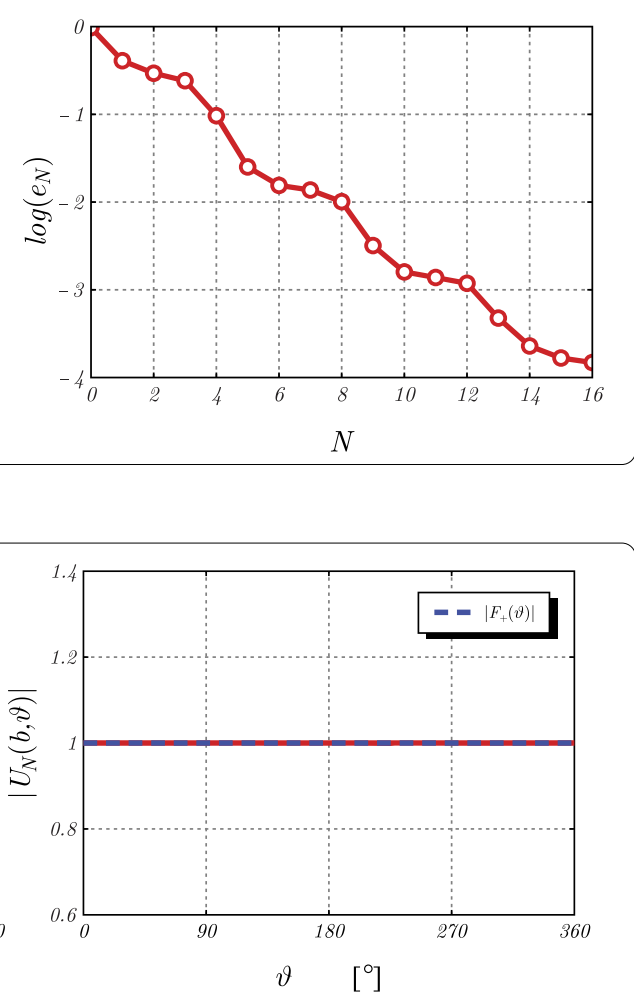

(b)

Figure 6 Boundary behavior along $\partial_{-} \mathcal{A}(a)$ and $\partial_{+} \mathcal{A}(b)$ of the partial sum $U_{N}(\varrho, \vartheta)$ of order $N=16$ representing the solution of the Dirichlet problem for the Laplace equation in the supershaped annulus $\mathcal{A}$ described by the Gielis formula with parameters $k_{x}^{-}=k_{y}^{-}=2, d_{x}^{-}=1 / 2, d_{y}^{-}=1, v_{x}^{-}=2$, $v_{y}^{-}=v_{0}^{-}=4, k_{x}^{+}=k_{y}^{+}=k^{+}=4, d_{x}^{+}=d_{y}^{+}=7 / 4$, and $v_{x}^{+}=v_{y}^{+}=2\left(1-v_{0}^{+} \log _{2} \cos \frac{\pi}{k^{+}}\right), v_{0}^{+} \rightarrow+\infty$.

Figure 7 Spatial distribution of the Fourier-like series expansion $v_{N}(x, y)$ of order $N=16$ representing the solution of the Dirichlet problem for the Laplace equation in the supershaped annulus $\mathcal{A}$ described by the Gielis formula with parameters $k_{x}^{-}=k_{y}^{-}=2, d_{x}^{-}=1 / 2, d_{y}^{-}$ $=1, v_{x}^{-}=2, v_{y}^{-}=v_{0}^{-}=4, k_{x}^{+}=k_{y}^{+}=k^{+}=4, d_{x}^{+}=d_{y}^{+}$ $=7 / 4$, and $v_{x}^{+}=v_{y}^{+}=2\left(1-v_{0}^{+} \log _{2} \cos \frac{\pi}{k^{+}}\right)$, $v_{0}^{+} \rightarrow+\infty$.

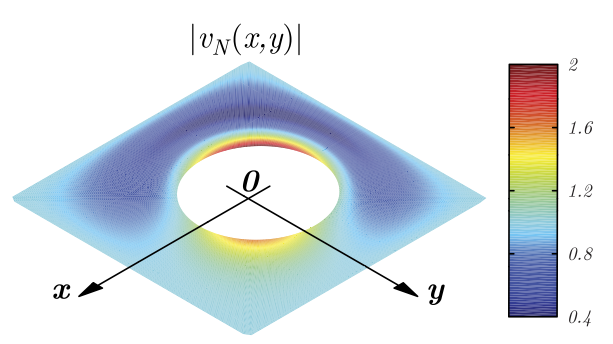

Figure 6, the selection of the expansion order $N=16$ results in an extremely accurate Fourier-like series representation $v_{N}(x, y)$ of the solution (with boundary error $e_{N}<0.1 \%$ ). The spatial distribution of $v_{N}(x, y)$ is shown in Figure 7, whereas the magnitude and phase of the relevant Fourier expansion coefficients $A_{m}, B_{m}$ are plotted in Figure 8.

Remark It has been observed that an $L^{2}$ norm of the difference between the exact solution and the relevant approximation is generally negligible. Point-wise convergence seems to be verified in the considered domains, with the only exception of a set of measure zero consisting of quasi-cusped points. In the neighborhood of these points, oscillations of the truncated order solution, recalling the classical Gibbs phenomenon, usually take place. 


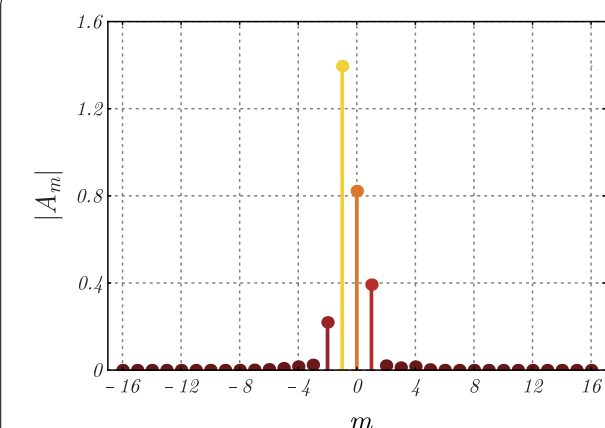

$(a)$

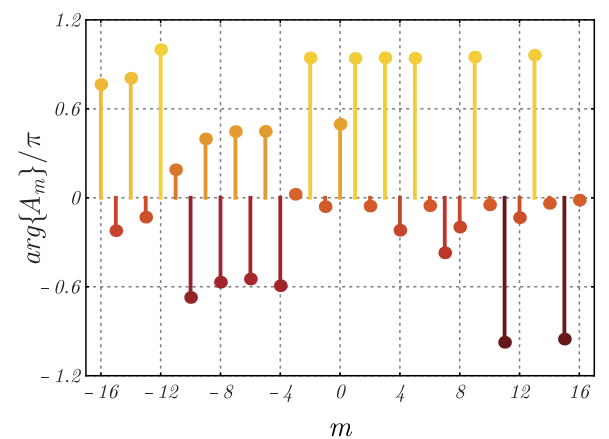

$(c)$

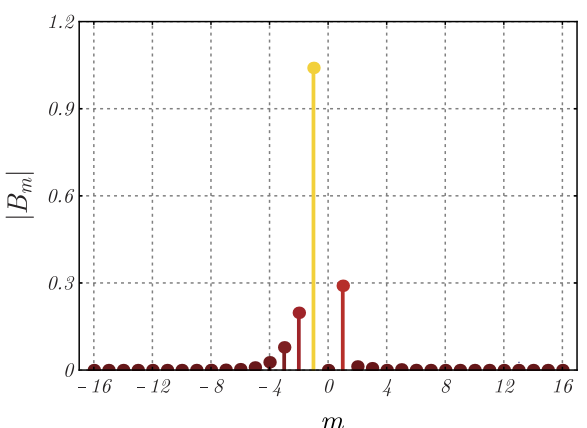

(b)

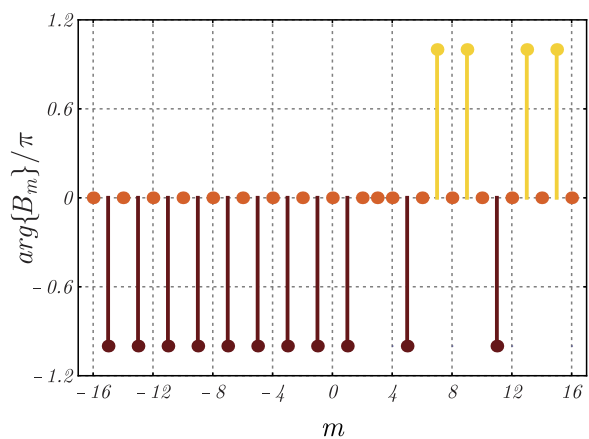

(d)

Figure 8 Magnitude (a), (b) and phase (c), (d) of the coefficients $A_{m}$ and $B_{m}$ relevant to the expansion $v_{N}(x, y)$ of order $N=16$ representing the solution of the Dirichlet problem for the Laplace equation in the supershaped annulus $\mathcal{A}$ described by the Gielis formula with parameters $k_{x}^{-}=k_{y}^{-}=2, d_{x}^{-}=1 / 2$, $d_{y}^{-}=1, v_{x}^{-}=2, v_{y}^{-}=v_{0}^{-}=4, k_{x}^{+}=k_{y}^{+}=k^{+}=4, d_{x}^{+}=d_{y}^{+}=7 / 4$, and $v_{x}^{+}=v_{y}^{+}=2\left(1-v_{0}^{+} \log _{2} \cos \frac{\pi}{k^{+}}\right)$, $v_{0}^{+} \rightarrow+\infty$.

\section{Conclusion}

A Fourier-like projection method, in combination with the adoption of a suitable stretched coordinate system, has been developed for solving the Dirichlet problem for the Laplace equation in supershaped annuli. In this way, analytically based expressions of the solution of the considered class of $B V P$ s can be derived by using classical quadrature rules, thus overcoming the need for cumbersome numerical techniques such as finite-difference or finite-element methods. The proposed approach has been successfully validated by means of a dedicated numerical procedure based on the computer-aided algebra tool Mathematica ${ }^{\odot}$. A point-wise convergence of the expansion series representing the solution seems to be verified with the only exception of a set of measure zero consisting of the quasi-cusped points along the boundary of the problem domain. In these points, Gibbslike oscillations may occur. The computed results are found to be in good agreement with the theoretical findings on Fourier series.

Competing interests

The authors declare that they have no competing interests.

Authors' contributions

DC proved the main theorem regarding the solution of the Laplace equation in supershaped annuli and drafted the paper. JG carried out the verification of the methodology and its application to Gielis domains. IT performed the numerical examples. PER derived the analytical expression of the Laplacian operator in stretched coordinates and helped to draft the manuscript. All authors read and approved the final manuscript. 


\section{Author details}

${ }^{1}$ Microwave Sensing, Signals and Systems, Delft University of Technology, Delft, The Netherlands. ${ }^{2}$ Department of Bioscience Engineering, University of Antwerp, Antwerp, Belgium. ${ }^{3}$ Faculty of Exact and Natural Sciences, Tbilisi State University, Tbilisi, Georgia. ${ }^{4}$ Faculty of Engineering, Campus Bio-Medico University, Rome, Italy.

\section{Acknowledgements}

Dedicated to Professor Hari M Srivastava

This research has been carried out under the grant PRIN/2006 Cap. 7320.

Received: 20 December 2012 Accepted: 17 April 2013 Published: 3 May 2013

\section{References}

1. Gakhov, FD: Boundary Value Problems. Dover, New York (1990)

2. Natalini, P, Patrizi, R, Ricci, PE: The Dirichlet problem for the Laplace equation in a starlike domain of a Riemann surface. Numer. Algorithms 28, 215-227 (2001)

3. Caratelli, D, Ricci, PE: The Dirichlet problem for the Laplace equation in a starlike domain. In: Proceedings of the International Conference on Scientific Computing, Las Vegas, 14-17 July 2008, pp. 160-166 (2008)

4. Caratelli, D, Germano, B, Gielis, J, He, MX, Natalini, P, Ricci, PE: Fourier Solution of the Dirichlet Problem for the Laplace and Helmholtz Equations in Starlike Domains. Lecture Notes of Tbilisi International Centre of Mathematics and Informatics. Tbilisi University Press, Tbilisi (2010)

5. Caratelli, D, Natalini, P, Ricci, PE, Yarovoy, A: The Neumann problem for the Helmholtz equation in a starlike planar domain. Appl. Math. Comput. 216, 556-564 (2010)

6. Caratelli, D, Gielis, J, Natalini, P, Ricci, PE, Tavkelidze, I: The Robin problem for the Helmholtz equation in a starlike planar domain. Georgian Math. J. 18, 465-480 (2011)

7. Caratelli, D, Gielis, J, Ricci, PE: Fourier-like solution of the Dirichlet problem for the Laplace equation in k-type Gielis domains. J. Pure Appl. Math., Adv. Appl. 5, 99-111 (2011)

8. Caratelli, D, Ricci, PE, Gielis, J: The Robin problem for the Laplace equation in a three-dimensional starlike domain. Appl. Math. Comput. 218, 713-719 (2011)

9. Gielis, J, Caratelli, D, Fougerolle, Y, Ricci, PE, Gerats, T: Universal natural shapes from unifying shape description to simple methods for shape analysis and boundary value problems. PLoS ONE (2012). doi:10.1371/journal.pone.0029324

10. Tolstov, GP: Fourier Series. Dover, New York (1962)

11. Lebedev, NN: Special Functions and Their Applications. Dover, New York (1972)

12. Gielis, J: A generic geometric transformation that unifies a wide range of natural and abstract shapes. Am. J. Bot. 90, 333-338 (2003)

13. Carleson, L: On convergence and growth of partial sums of Fourier series. Acta Math. 116, 135-157 (1966)

14. Lenjou, K: Krommen en oppervlakken van Lamé and Gielis. MSc thesis, Catholic University of Leuven (2005)

doi:10.1186/1687-2770-2013-113

Cite this article as: Caratelli et al.: The Dirichlet problem for the Laplace equation in supershaped annuli. Boundary Value Problems 2013 2013:113.

\section{Submit your manuscript to a SpringerOpen ${ }^{\ominus}$ journal and benefit from:}

- Convenient online submission

Rigorous peer review

Immediate publication on acceptance

Open access: articles freely available online

- High visibility within the field

- Retaining the copyright to your article 